\title{
Separation of bulk and interface contributions to electroforming and resistive switching behavior of epitaxial Fe-doped $\mathrm{SrTiO}_{3}$
}

T. Menke, P. Meuffels, R. Dittmann, K. Szot, and R. Waser

Citation: Journal of Applied Physics 105, 066104 (2009);

View online: https://doi.org/10.1063/1.3100209

View Table of Contents: http://aip.scitation.org/toc/jap/105/6

Published by the American Institute of Physics

\section{Articles you may be interested in}

Impact of the electroforming process on the device stability of epitaxial Fe-doped $\mathrm{SrTiO}_{3}$ resistive switching cells Journal of Applied Physics 106, 114507 (2009); 10.1063/1.3267485

Characteristic electroforming behavior in $\mathrm{Pt} / \mathrm{TiO}_{2} / \mathrm{Pt}$ resistive switching cells depending on atmosphere Journal of Applied Physics 104, 123716 (2008); 10.1063/1.3043879

Resistive switching mechanism of $\mathrm{TiO}_{2}$ thin films grown by atomic-layer deposition

Journal of Applied Physics 98, 033715 (2005); 10.1063/1.2001146

Field-induced resistive switching in metal-oxide interfaces

Applied Physics Letters 85, 317 (2004); 10.1063/1.1768305

Resistive switching phenomena: A review of statistical physics approaches

Applied Physics Reviews 2, 031303 (2015); 10.1063/1.4929512

Electro-degradation and resistive switching of Fe-doped $\mathrm{SrTiO}_{3}$ single crystal Journal of Applied Physics 113, 083713 (2013); 10.1063/1.4793632

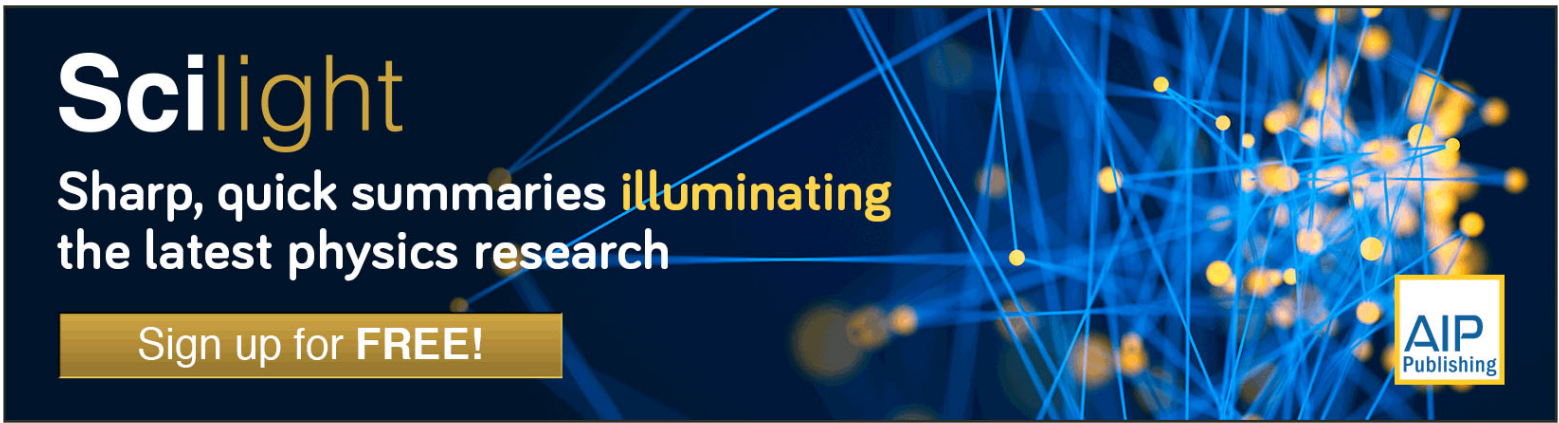




\title{
Separation of bulk and interface contributions to electroforming and resistive switching behavior of epitaxial Fe-doped $\mathrm{SrTiO}_{3}$
}

\author{
T. Menke, ${ }^{\text {a) }}$ P. Meuffels, R. Dittmann, K. Szot, and R. Waser \\ Institute of Solid State Research, Forschungszentrum Juelich, 52425 Juelich, Germany
}

(Received 16 February 2009; accepted 17 February 2009; published online 26 March 2009)

\begin{abstract}
We succeeded in the separation of bulk and interface contributions to the electroforming and resistive switching behavior of $\mathrm{Pt} / \mathrm{STO}(\mathrm{Fe}) / \mathrm{Nb}: \mathrm{STO}$ devices by performing impedance spectroscopy. Two distinctive features observed in the impedance spectra could be assigned to the $\mathrm{STO}(\mathrm{Fe})$ bulk and to the depletion layer of the Pt/STO $(\mathrm{Fe})$ Schottky contact. We attribute the resistance change during the dc forming process to a local bypassing of the depletion layer caused by oxygen effusion to the environment. By comparing the impedance spectra in the resistive "on" and "off" states, we propose that the resistance of the $\mathrm{STO}(\mathrm{Fe}) / \mathrm{Nb}: \mathrm{STO}$ interface locally changes during the switching process. (c) 2009 American Institute of Physics. [DOI: 10.1063/1.3100209]
\end{abstract}

For realizing a new solid-state memory technology, there is a strong interest in the application of transition metal oxides. ${ }^{1}$ Even though the exact microscopic mechanisms for bipolar resistive switching in these oxides are still under controversial discussion, there exists a general agreement that the migration of oxygen ions under an applied electric field plays a key role. ${ }^{2}$

Among the ternary oxides, $\mathrm{SrTiO}_{3}$ appears to be a good candidate as prototype material for the further elucidation of the microscopic switching mechanism. ${ }^{1}$ To enable the resistive switching properties in this band-gap insulator, generally a forming procedure is needed. By applying thermal ${ }^{3}$ or electrical stress, ${ }^{4}$ the conductivity can be drastically increased due to self-doping induced by the formation of oxygen vacancies. The location of the forming and the switching phenomena within metal/insulator/metal (MIM) structures has been investigated by using lateral electrode configurations and it has been shown that the switching of the resistance is mainly located at the metal-insulator interface. ${ }^{5}$

To close the link between capacitive and lateral MIM structures and to get a deeper understanding of the forming and switching mechanism, we studied the electrical properties in capacitorlike structures of epitaxial grown Fe-doped $\mathrm{SrTiO}_{3}$ thin films by impedance spectroscopy.

The investigated epitaxial 1 at. \% Fe-doped $\mathrm{SrTiO}_{3}$ $[\mathrm{STO}(\mathrm{Fe})]$ thin films with thicknesses between 50 and 750 $\mathrm{nm}$ were grown by pulsed laser deposition. The films were deposited on (100)-oriented 1 at. \% Nb-doped $\mathrm{SrTiO}_{3}(\mathrm{Nb}$ :STO) single crystals serving both as a substrate and as a bottom electrode for the MIM structures. Further details of the deposition process can be extracted from Ref. 6. To complete the MIM structure, $100 \mathrm{~nm}$ Pt top electrodes were deposited and patterned to electrode diameters of $200 \mu \mathrm{m}$.

Impedance spectroscopy was performed in the frequency range $0.1 \mathrm{~Hz}-3 \mathrm{MHz}$ using a Solatron impedance analyzer in combination with a preamplifier. All impedance measurements were taken at $50 \mathrm{mV}_{\text {rms }}$.

\footnotetext{
${ }^{a)}$ Electronic mail: t.menke@fz-juelich.de.
}

The $I-V$ curves of pristine samples showed rectifying characteristics which we assign to the Schottky contact formed at the interface between the high workfunction metal Pt $(\phi: 5.65 \mathrm{eV})$ and the STO(Fe) film.

Even though the last few monolayers of the $\mathrm{STO}(\mathrm{Fe})$ film might show some deviations from stoichiometry due to the Pt deposition, ${ }^{7}$ we will discuss the properties of this junction by means of the Schottky model. It cannot be excluded that the depletion layer may also result from a Bardeen barrier due to a high concentration of surface states. This, however, would not change our principal conclusions.

The forward direction of this blocking contact was obtained by applying a positive voltage bias to the Pt top electrode. To justify the assumption of a Pt/STO $(\mathrm{Fe})$ Schottky contact, a reference sample using a metal with a shallow workfunction as the top electrode, in this case $\mathrm{Ti}$ $(\phi: 4.3 \mathrm{eV})$, was prepared. This sample exhibited a symmetric $I-V$ characteristic as well as a significant lower device resistance (not shown here), which demonstrate the ohmic nature of both $\mathrm{Ti} / \mathrm{STO}(\mathrm{Fe})$ and $\mathrm{STO}(\mathrm{Fe}) / \mathrm{Nb}$ :STO interfaces. From the results of both types of samples, we can conclude that even though the $\mathrm{STO}(\mathrm{Fe})$ thin films are nominally acceptor doped, they exhibit a significant $n$-type conductivity at room temperature which we attribute to the self-doping by oxygen vacancies formed during the deposition procedure.

If the film thickness is larger than the width of the Pt/ $\mathrm{STO}(\mathrm{Fe})$ related Schottky depletion layer, one expects two capacitive effects (bulk and depletion layer) ${ }^{8}$ contributing to the frequency dependent complex impedance. We determined the depletion layer thickness by typical $C(V)$ measurement to be $\sim 200 \mathrm{~nm}$ for our material system. This was confirmed for samples with thicknesses smaller than the expected depletion layer width where only one semicircle could be observed in the complex impedance plane.

We measured the impedance spectra of a $750 \mathrm{~nm}$ thick $\mathrm{STO}(\mathrm{Fe})$ sample in the pristine, unformed state. The corresponding Cole-Cole plot and the equivalent circuit which is proposed to fit the data are shown in Fig. 1(a) and in the inset (i), respectively. The series inductance of the leads as well as the series resistance of the $\mathrm{Nb}$ :STO bottom electrode were 

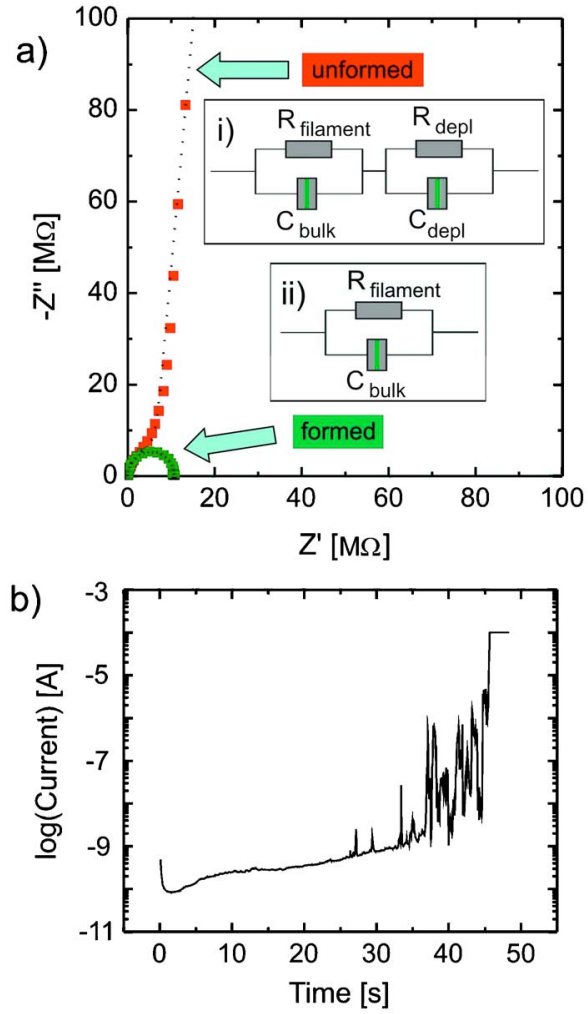

FIG. 1. (Color online) (a) Impedance spectra of a Pt/STO(Fe)/Nb:STO MIM structure before and after a dc forming process. The equivalent circuits used for fitting are displayed in the inset for (i) the unformed case with $R_{\text {_filament }}=7.3 \mathrm{M} \Omega, C_{\text {_bulk }}=277 \mathrm{pF}, R_{\text {depl }}=17.9 \mathrm{G} \Omega$, and $C_{\text {_depl }}=914 \mathrm{pF}$ and for (ii) the formed case with $R_{\text {filament }}=10.7 \mathrm{M} \Omega$ and $C_{\text {bulk }}=273 \mathrm{pF}$. (b) Related $I-t$ curve during electroforming recorded by applying $+10 \mathrm{~V}$ to the Pt top electrode. The spike-like fluctuations will be attributed to the reduction and reoxidation of filament fragments.

taken into account for fitting the equivalent circuit but are not depicted in the diagrams. The impedance spectra of the $\mathrm{STO}(\mathrm{Fe})$ film can be perfectly described by two Voigt elements resulting from the bulk and the depletion layer properties.

By means of measurements with an atomic force microscope equipped with a conducting tip, the filamentary conduction of our $\mathrm{STO}(\mathrm{Fe})$ thin films could be proved. This is in agreement with the results reported in literature for single crystals and thin films. ${ }^{9}$ Thus, the bulk related contribution to the impedance of the thick films has to be described by conducting filaments in a more or less insulating matrix. The bulk related Voigt element should therefore exhibit two different resistances in parallel to account for the conductance inhomogeneity. Unfortunately, there is no possibility to divide between both resistances by impedance measurements, since the bulk conductivity $1 / R$ will be dominated by the conductivity of the filaments $\left(1 / R=1 / R_{\text {matrix }}+1 / R_{\text {filament }}\right.$ $\left.\approx 1 / R_{\text {filament }}\right)$.

To attribute the Voigt elements to their physical origin, dc-bias dependent impedance measurements were performed. It is well known that the bulk impedance should only be slightly influenced by an applied dc bias, whereas the depletion layer impedance should clearly vary with an applied dc bias. ${ }^{10}$ This was indeed found for our samples. Ad- ditionally, the film thickness dependence of both elements showed a scaling of the bulk capacitance and a thickness independence of the depletion layer capacitance so that the two Voigt elements could be clearly allocated. This analysis implies that the overall resistance is dominated by the depletion layer resulting from the Schottky contact at the Pt/ $\mathrm{STO}(\mathrm{Fe})$ interface.

Unlike to samples with a low initial resistance which do not need any forming treatment, ${ }^{6}$ the investigated devices with Pt electrodes require an electroforming step setting the sample to the low resistance state (LRS). For this purpose, a dc voltage of $+10 \mathrm{~V}$ was applied to the Pt electrode, corresponding to the forward direction of the Schottky contact. The resulting current-time (I-t) curve is shown in Fig. 1(b). During the first $25 \mathrm{~s}$, the current remains in the subnanoampere regime and increases just slightly with the time.

After $45 \mathrm{~s}$, a steep increase in the current occurs and the current compliance, which was set to $100 \mu \mathrm{A}$ to prevent the sample from electrical breakdown, is attained. As soon as the current reached the compliance, we stopped the forming process because, according to our experience, at this stage bipolar switching could be observed. The result of the impedance measurements on the formed pad can be seen in Fig. 1(a) and can be described using the equivalent circuit displayed in the inset (ii). The most significant change is that in the case of a formed sample only one Voigt element is needed to fit the data. As the values for the resistance and the capacitance are comparable to the values obtained for the bulk related Voigt element in the unformed state, we attribute the Voigt element in the formed state to bulk contribution.

In order to understand our experimental observations, we have to consider that electroforming removes oxygen ions from the STO lattice which becomes evident due to the appearance of gas bubbles under the anode. ${ }^{1}$ By applying a positive bias to the top electrode, oxygen ions migrate along the extended defects toward the top interface and are released to the surrounding. ${ }^{11}$ In contrast to the STO single crystals, where a virtual cathode moves toward the anode until a conductive path is established during the forming procedure, ${ }^{12}$ our as-deposited thin films already contain preformed conducting paths as a result of the reducing deposition conditions.

Since the overall resistance in the unformed case is dominated by the depletion layer, most of the applied voltage drops in the vicinity of the top interface. Taking into account that the movement of oxygen ions is a field-driven process, we conclude that the electroforming process most likely removes oxygen directly next to the top interface where the electrical field is strongly enhanced. Due to the donor character of oxygen vacancies, a significant reduction in the effective Schottky barrier height and of the depletion layer width at the $\mathrm{Pt} / \mathrm{STO}(\mathrm{Fe})$ interface can be expected. This is in agreement with the voltage-induced elimination of the Schottky barrier observed at the interface between Pt and $\mathrm{TiO}_{2}$ single crystals. ${ }^{13}$

In order to clarify whether electroforming was a local or a homogenous effect, an additional experiment was performed. After a successful electroforming step at a certain MIM device, the electrode pad was divided into two parts 

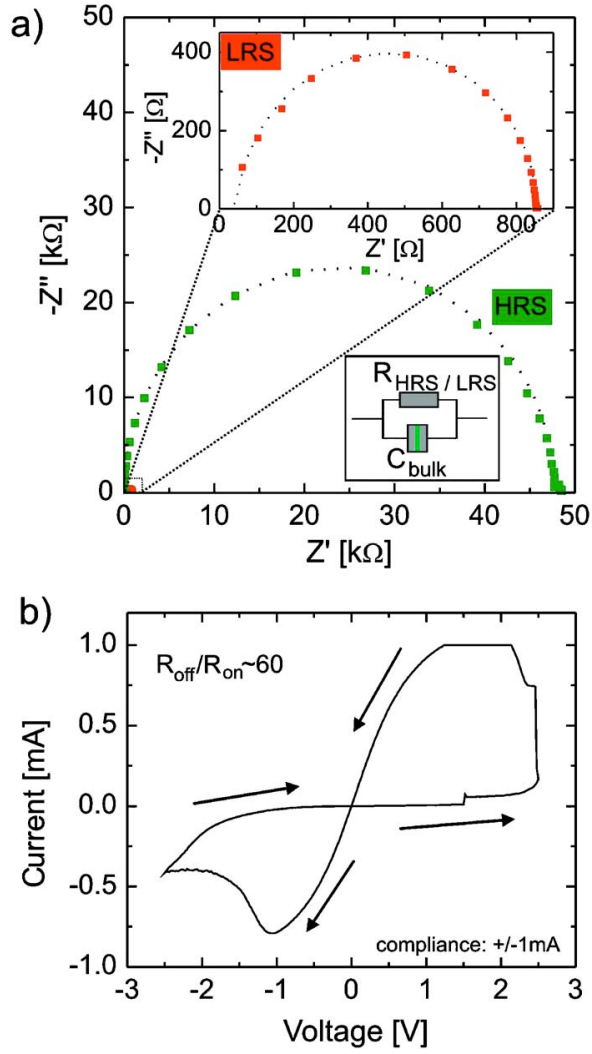

FIG. 2. (Color online) (a) Impedance spectra of a Pt/STO(Fe)/Nb:STO MIM structure in two different resistive states. The corresponding equivalent circuit is depicted in the inset. The $I-V$ curve displayed in part (b) shows a typical bipolar resistive switching hysteresis of a Pt/STO $(\mathrm{Fe}) / \mathrm{Nb}: \mathrm{STO}$ stack.

and each part of the device was measured again. Whereas one part showed the reduced resistance which was measured before on the whole electroformed device, the other part was in the initial high-ohmic state. This experiment clearly points to the local nature of the electroforming process. This localized bypassing of the Schottky depletion layer explains the vanishing of the complete contribution of the depletion layer to the impedance and also that the bulk properties are not significantly changed during the forming procedure.

To confirm this statement a reference sample with top electrodes of Ti providing an ohmic contact was prepared. As expected, the impedance spectra exhibited only the bulk contribution and no electroforming process was necessary to achieve bipolar resistive switching (not shown here).

The typical switching behavior of $\mathrm{Pt} / \mathrm{STO}(\mathrm{Fe})$ samples treated with the above described forming procedure can be seen in the $I$ - $V$ curve of Fig. 2(b). Switching the pad to the high resistance state (HRS) was achieved by applying a voltage sweep from 0 to $-2.5 \mathrm{~V}$ and back to $0 \mathrm{~V}$. In this part of the $I-V$ characteristic, a negative differential resistance arises during sweeping up to $-2.5 \mathrm{~V}$ which is a sufficient condition for bipolar resistive switching. After finishing the negative sweep, the resistance measured at $50 \mathrm{mV}$ increased almost by a factor of 60 . For a further investigation of the resistive states, impedance measurements were performed in the LRS and HRS, which were set by positive or negative voltage sweeps. The corresponding Cole-Cole plots, shown in Fig. 2(a), can be described by using equivalent circuits consisting of only one Voigt element. A comparison of the fitted values showed that the device capacitance in both states (LRS: 284 pF; HRS: $265 \mathrm{pF}$ ) remained nearly unchanged. Unlike to the capacitance, the resistance changed from $828 \Omega$ in the LRS to $47.4 \mathrm{k} \Omega$ in the HRS. It has to be pointed out that the LRS as well as the HRS can be described without taking into account the depletion layer influence. This implies that "opening and closing" of the conducting channels during the switching process is not located at the $\mathrm{Pt} / \mathrm{STO}(\mathrm{Fe})$ interface due to the fact that this would lead to a restoring of the, only locally shunted, depletion layer impedance.

The capability of this material class to switch in the nanosecond regime and the results from lateral measurements ${ }^{4}$ lead to the assumption that the origin of the switching is localized at an interface. The absence of the depletion layer impedance especially in the HRS indicates that in our case the $\mathrm{STO}(\mathrm{Fe}) / \mathrm{Nb}$ :STO interface is the relevant one for the switching behavior. We suggest that a local variation in the transfer resistance at $\mathrm{STO}(\mathrm{Fe}) / \mathrm{Nb}: \mathrm{STO}$ interface resulting from a redistribution of oxygen vacancies via conducting filament is the origin of this resistance change. Further details of the microscopic mechanisms at the bottom interface are subject of our ongoing research.

In conclusion, we presented that a dc forming process removes oxygen along the extended defects in the vicinity of the Pt/STO $(\mathrm{Fe})$ interface thus bypassing the Schottky depletion layer. Bipolar resistive switching was achieved by applying a voltage sweep leading to a $R_{\text {off }} / R_{\text {on }}$ ratio of about 60. During the bipolar resistive switching, the bulk contribution of the impedance can be varied and this may be explained by the oxygen related opening/closing of conducting channels at the $\mathrm{STO}(\mathrm{Fe}) / \mathrm{Nb}: \mathrm{STO}$ interface.

We thank R. Muenstermann and K. Shibuya for helpful discussions and Intel Inc. for financial support.

${ }^{1}$ K. Szot, W. Speier, G. Bihlmayer, and R. Waser, Nature Mater. 5, 312 (2006).

${ }^{2}$ R. Waser and M. Aono, Nature Mater. 6, 833 (2007); A. Sawa, Mater. Today 11, 28 (2008).

${ }^{3}$ K. Szot, W. Speier, R. Carius, U. Zastrow, and W. Beyer, Phys. Rev. Lett. 88, 075508 (2002).

${ }^{4}$ M. Janousch, G. I. Meijer, U. Staub, B. Delley, S. F. Karg, and B. P. Andreasson, Adv. Mater. (Weinheim, Ger.) 19, 2232 (2007).

${ }^{5}$ J. J. Yang, M. D. Pickett, X. Li, D. A. A. Ohlberg, D. R. Stewart, and R. S. Williams, Nat. Nanotechnol. 3, 429 (2008).

${ }^{6}$ R. Oligschlaeger, R. Waser, R. Meyer, S. Karthauser, and R. Dittmann, Appl. Phys. Lett. 88, 042901 (2006); W. Shen, R. Dittmann, U. Breuer, and R. Waser, ibid. 93, 222102 (2008).

${ }^{7}$ B. Psiuk, J. Szade, H. Schroeder, H. Haselier, M. Mlynarczyk, R. Waser, and K. Szot, Appl. Phys. A: Mater. Sci. Process. 89, 451 (2007).

${ }^{8}$ J. Balaya, J. Jamnik, J. Fleig, and J. Maier, J. Electrochem. Soc. 154, P69 (2007).

${ }^{9}$ K. Szot, R. Dittmann, W. Speier, and R. Waser, Phys. Status Solidi (RRL) 1, R86 (2007); C. Rossel, G. I. Meijer, D. Bremaud, and D. Widmer, J. Appl. Phys.. 90, 2892 (2001); R. Muenstermann, R. Dittmann, K. Szot, S. Mi, C. L. Jia, P. Meuffels, and R. Waser, Appl. Phys. Lett. 93, 023110 (2008).

${ }^{10}$ I. Denk, J. Claus, and J. Maier, J. Electrochem. Soc. 144, 3526 (1997).

${ }^{11}$ D. S. Jeong, H. Schroeder, and R. Waser, J. Appl. Phys. 104, 123716 (2008).

${ }^{12}$ T. Baiatu, R. Waser, and K. H. Hardtl, J. Am. Ceram. Soc. 73, 1663 (1990).

${ }^{13}$ J. R. Jameson, Y. Fukuzumi, Z. Wang, P. Griffin, K. Tsunoda, G. I. Meijer, and Y. Nishi, Appl. Phys. Lett. 91, 112101 (2007). 